\title{
A generalization of a combinatorial identity by Chang and Xu
}

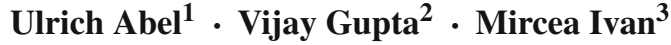

Received: 30 December 2014 / Revised: 31 July 2015 / Accepted: 3 August 2015 /

Published online: 15 September 2015

(C) The Author(s) 2015. This article is published with open access at SpringerLink.com

\begin{abstract}
Recently, Chang and Xu gave a probabilistic proof of a combinatorial identity which involves binomial coefficients. Duarte and Guedes de Oliveira (J Integer Seq 16, 2013) extended the result. Applying a generalization of the Leibniz rule for higher derivatives of the product of functions yields a new short proof and a generalization of the above mentioned identity.
\end{abstract}

Keywords Combinatorial identities

Mathematics Subject Classification 05A19

Communicated by S.K. Jain.

Ulrich Abel

ulrich.abel@mnd.thm.de

Vijay Gupta

vijaygupta2001@hotmail.com

Mircea Ivan

Mircea.Ivan@math.utcluj.ro

1 Department MND, Technische Hochschule Mittelhessen, Wilhelm-Leuschner-Straße 13, 61169 Friedberg, Germany

2 Department of Mathematics, Netaji Subhas Institute of Technology, Sector 3 Dwarka, New Delhi 110078, India

3 Department of Mathematics, Technical University of Cluj-Napoca, Str. Memorandumului nr. 28, 400114 Cluj-Napoca, Romania 


\section{Introduction and main results}

Throughout the paper $\mathbf{k}=\left(k_{1}, \ldots, k_{r}\right) \in \mathbb{N}_{0}^{r}$ denotes a multi-index, $|\mathbf{k}|=k_{1}+\cdots+k_{r}$, and the multinomial coefficient is defined by

$$
\left(\begin{array}{l}
n \\
\mathbf{k}
\end{array}\right)=\left(\begin{array}{c}
n \\
k_{1}, \ldots, k_{r}
\end{array}\right):=\frac{n !}{k_{1} ! \ldots k_{r} !(n-|\mathbf{k}|) !} .
$$

In 2011, Chang and Xu [4, Theorem 1] gave a probabilistic proof of the combinatorial identity

$$
\sum_{|\mathbf{k}|=n}\left[\prod_{i=1}^{r}\left(\begin{array}{c}
2 k_{i} \\
k_{i}
\end{array}\right)\right]=4^{n}\left(\begin{array}{c}
n+r / 2-1 \\
n
\end{array}\right),
$$

for $n, r \in \mathbb{N}, r \geq 2$, which involves central binomial coefficients. It consists of showing that the identity essentially computes the moment of order $n$ of the chi-square random variable with $r$ degrees of freedom. The special instance $r=2$, i.e.,

$$
\sum_{k=0}^{n}\left(\begin{array}{c}
2 k \\
k
\end{array}\right)\left(\begin{array}{c}
2(n-k) \\
n-k
\end{array}\right)=4^{n}
$$

plays an important role in combinatorics and probability theory (see the introduction of [4]). In 2013, Duarte and Guedes de Oliveira [2, Theorem 2] showed that, for $\mathbf{a}=\left(a_{1}, \ldots, a_{r}\right) \in \mathbb{R}^{r}$ with $|\mathbf{a}|=0$,

$$
\sum_{|\mathbf{k}|=n}\left[\prod_{i=1}^{r}\left(\begin{array}{c}
2 k_{i}-a_{i} \\
k_{i}
\end{array}\right)\right]=4^{n}\left(\begin{array}{c}
n+r / 2-1 \\
n
\end{array}\right) .
$$

The special case $r=2$, reveals that, for all constants $a \in \mathbb{R}$,

$$
\sum_{k=0}^{n}\left(\begin{array}{c}
2 k-a \\
k
\end{array}\right)\left(\begin{array}{c}
2(n-k)+a \\
n-k
\end{array}\right)=4^{n}
$$

where the sum in the left-hand side is independent of a. Identities for similar sums can be found in [3, Theorem 1].

We study the identity (1) without the restriction $|\mathbf{a}|=0$. In particular, we give a presentation of the sum

$$
\sum_{|\mathbf{k}|=n}\left[\prod_{i=1}^{r}\left(\begin{array}{c}
c k_{i}-a_{i} \\
k_{i}
\end{array}\right)\right]
$$

as a derivative of an explicitly defined function, for arbitrary $c \in \mathbb{R}$ and $\mathbf{a}=$ $\left(a_{1}, \ldots, a_{r}\right) \in \mathbb{R}^{r}$. In the special case $c=2$ and when it happens that $|\mathbf{a}|$ is a nonnegative integer, this sum can be represented in terms of a sum consisting of $|\mathbf{a}|+1$ 
summands. While Chang and Xu used probabilistic arguments involving the expected value of a $\chi^{2}$ random variable, and Duarte and Guedes de Oliveira used standard combinatorial tools like generating functions, our proof is based on methods of complex analysis.

The main result is the following identity.

Theorem 1 For $n, r \in \mathbb{N}, r \geq 2$, and $\mathbf{a}=\left(a_{1}, \ldots, a_{r}\right) \in \mathbb{R}^{r}$ such that $|\mathbf{a}|$ is a nonnegative integer,

$$
\sum_{|\mathbf{k}|=n}\left[\prod_{i=1}^{r}\left(\begin{array}{c}
2 k_{i}-a_{i} \\
k_{i}
\end{array}\right)\right]=4^{n-|a| / 2} \sum_{j=0}^{|a|}\left(\begin{array}{c}
|\mathbf{a}| \\
j
\end{array}\right)\left(\begin{array}{c}
n+(r-|\mathbf{a}|+j) / 2-1 \\
n
\end{array}\right) .
$$

Remark 1 In the special case $|\mathbf{a}|=0$ Theorem 1 reduces to the identity (1) by Duarte and Guedes de Oliveira.

Corollary 1 Under the assumptions of Theorem 1 , the condition $|\mathbf{a}|=0$ implies that

$$
\sum_{|\mathbf{k}|=n}\left[\prod_{i=1}^{r}\left(\begin{array}{c}
2 k_{i}-a_{i} \\
k_{i}
\end{array}\right)\right]=4^{n}\left(\begin{array}{c}
n+r / 2-1 \\
n
\end{array}\right) .
$$

Proposition 1 For $n, r \in \mathbb{N}, r \geq 2, c \in \mathbb{R}$ and $\mathbf{a}=\left(a_{1}, \ldots, a_{r}\right) \in \mathbb{R}^{r}$,

$$
\sum_{|\mathbf{k}|=n}\left[\prod_{i=1}^{r}\left(\begin{array}{c}
c k_{i}-a_{i} \\
k_{i}
\end{array}\right)\right]=\frac{1}{n !} F_{n}^{(n)}(0)
$$

where

$$
F_{n}(x) \equiv F_{n}(r, c, \mathbf{a} ; x):=\frac{(1+x)^{c n+r-1-|\mathbf{a}|}}{(1+(1-c) x)^{r-1}} .
$$

Remark 2 Application of the Leibniz Rule for the derivatives of products of differentiable functions implies as an immediate consequence the formula

$$
\sum_{|\mathbf{k}|=n}\left[\prod_{i=1}^{r}\left(\begin{array}{c}
c k_{i}-a_{i} \\
k_{i}
\end{array}\right)\right]=\sum_{k=0}^{n}\left(\begin{array}{c}
c n-|\mathbf{a}|+r-1 \\
k
\end{array}\right)\left(\begin{array}{c}
r-2+n-k \\
n-k
\end{array}\right)(c-1)^{n-k}
$$

which reduces the number of multiplied binomial coefficients from $r$ to two.

Recently, Michael Z. Spivey gave a combinatorial proof of the alternating sum

$$
\sum_{k=0}^{n}(-1)^{k}\left(\begin{array}{c}
2 k \\
k
\end{array}\right)\left(\begin{array}{c}
2(n-k) \\
n-k
\end{array}\right)= \begin{cases}2^{n}\left(\begin{array}{c}
n \\
n / 2
\end{array}\right), & n \text { even } \\
0, & n \text { odd }\end{cases}
$$

by applying an involution to certain colored permutations. 


\section{Proofs and auxiliary results}

The proofs of the results in the preceding section are essentially based on the following formula.

Lemma 1 Let $n \in \mathbb{N}_{0}, r \in \mathbb{N}$, and let $h, f_{i}(i=1, \ldots, r)$ be functions which are $n$ times differentiable in $x_{0} \in \mathbb{R}$ with $h\left(x_{0}\right) \neq 0$. Then

$$
\sum_{|\mathbf{k}|=n}\left(\begin{array}{l}
n \\
\mathbf{k}
\end{array}\right) \prod_{i=1}^{r}\left(h^{k_{i}} f_{i}\right)^{\left(k_{i}\right)}\left(x_{0}\right)=\left.\left(\left(\frac{\mathrm{d}}{\mathrm{d} x}\right)^{n} \frac{h^{n+r-1}(x) \prod_{i=1}^{r} f_{i}(x)}{\left(h(x)-h^{\prime}(x)\left(x-x_{0}\right)\right)^{r-1}}\right)\right|_{x=x_{0}} .
$$

A proof of the intriguing formula (4) can be found in [1]. If $h$ is a constant function, Eq. (4) obviously reduces to the well-known Leibniz Rule

$$
\left(\prod_{i=1}^{r} f_{i}\right)^{(n)}=\sum_{|\mathbf{k}|=n}\left(\begin{array}{l}
n \\
\mathbf{k}
\end{array}\right) \prod_{i=1}^{r} f_{i}{ }^{\left(k_{i}\right)} \quad(n=0,1,2, \ldots),
$$

for several $n$ times differentiable functions $f_{i}(i=1, \ldots, r)$.

Proof of Prop. 1. We put

$$
\begin{aligned}
& h(x)=x^{c}, \\
& f_{i}(x)=x^{-a_{i}}, \quad \text { for } 1 \leq i \leq r .
\end{aligned}
$$

The left-hand side of Eq. (4) is equal to

$$
\begin{aligned}
\text { LHS } & =\sum_{|\mathbf{k}|=n}\left(\begin{array}{l}
n \\
\mathbf{k}
\end{array}\right) \prod_{i=1}^{r}\left(h^{k_{i}} f_{i}\right)^{\left(k_{i}\right)}\left(x_{0}\right) \\
& =n ! x_{0}^{(c-1) n-|\mathbf{a}|} \sum_{|\mathbf{k}|=n}\left[\prod_{i=1}^{r}\left(\begin{array}{c}
c k_{i}-a_{i} \\
k_{i}
\end{array}\right)\right] .
\end{aligned}
$$

The right-hand side of Eq. (4) is equal to

$$
\operatorname{RHS}=\left.\left(\left(\frac{\mathrm{d}}{\mathrm{d} x}\right)^{n} \frac{x^{c(n+r-1)-|\mathbf{a}|}}{\left((1-c) x+c x_{0}\right)^{r-1}}\right)\right|_{x=x_{0}},
$$

where we used that

$$
h(x)-h^{\prime}(x)\left(x-x_{0}\right)=x^{c-1}\left((1-c) x+c x_{0}\right) .
$$

Comparison of both sides with $x_{0}=1$ leads to

$$
n ! \sum_{|\mathbf{k}|=n} \prod_{i=1}^{r}\left(\begin{array}{c}
c k_{i}-a_{i} \\
k_{i}
\end{array}\right)=\left.\left(\left(\frac{\mathrm{d}}{\mathrm{d} x}\right)^{n} \frac{x^{c n+r-1-|\mathbf{a}|}}{((1-c) x+c)^{r-1}}\right)\right|_{x=1}
$$

which implies the assertion. 
Proof of Theorem 1. Put $c=2$ in Prop. 1. Taking advantage of the Cauchy integral formula we obtain, for the function $F$ as defined in (3),

$$
F_{n}^{(n)}(0)=\frac{n !}{2 \pi i} \int_{W_{1}} \frac{(1+z)^{2 n-|\mathbf{a}|}}{z^{n+1}}\left(\frac{1+z}{1-z}\right)^{r-1} \mathrm{~d} z,
$$

where the integration path $W_{1}=\partial D_{\rho}=\{z|| z \mid=\rho\}$ with sufficiently small $\rho>0$ encircles the origin counterclockwise. The change of variables

$$
z=\frac{w-1}{w+1}, \quad \mathrm{~d} z=2(w+1)^{-2} \mathrm{~d} w
$$

i.e., $w=(1+z) /(1-z)$, yields

$$
F_{n}^{(n)}(0)=2^{2 n-|\mathbf{a}|+1} \frac{n !}{2 \pi i} \int_{W_{2}} \frac{w^{2 n-|\mathbf{a}|+r-1}(w+1)^{|\mathbf{a}|}}{\left(w^{2}-1\right)^{n+1}} \mathrm{~d} w
$$

where the integration path $W_{2}$ encounters $w_{0}=1$ such that $\operatorname{Re}(w+1)>0$. A second change of variables $w=\sqrt{5}$ yields

$$
F_{n}^{(n)}(0)=2^{2 n-|\mathbf{a}|} \frac{n !}{2 \pi i} \int_{W_{3}} \frac{\varsigma^{n+\frac{-|\mathbf{a}|+r}{2}-1}(\sqrt{\varsigma}+1)^{|\mathbf{a}|}}{(\varsigma-1)^{n+1}} \mathrm{~d} \varsigma,
$$

where the integration path $W_{3}$ encounters $\varsigma_{0}=1$ such that $\operatorname{Re} \varsigma>0$. When it happens that $|\mathbf{a}| \in \mathbb{N}_{0}$ expansion by the binomial formula and application of the Cauchy integral formula completes the proof of Theorem 1 .

\section{An alternative proof of Theorem 1}

In this section we present an alternative derivation of Theorem 1 . The referee pointed out that our main result (2) is a consequence of results by Duarte and Guedes de Oliveira [2] and suggested a proof. It is based on the technique of generating functions and will be outlined below. To this end denote, as in [2, Sect. 4], by

$$
g(x):=\frac{1}{\sqrt{1-4 x}}=\sum_{n \geq 0}\left(\begin{array}{c}
2 n \\
n
\end{array}\right) x^{n}
$$

and

$$
C(x):=\frac{2}{1+\sqrt{1-4 x}}=\sum_{n \geq 0} \frac{1}{n+1}\left(\begin{array}{c}
2 n \\
n
\end{array}\right) x^{n}
$$

the generating functions of the central binomial coefficients and of the Catalan numbers, respectively. By [2, Theorem 11], we have, for all real numbers $s$ and $t$, 


$$
g(x) C(x)^{s}=\sum_{n \geq 0}\left(\begin{array}{c}
2 n+s \\
n
\end{array}\right) x^{n}
$$

and

$$
g(x)^{t}=\sum_{n \geq 0} 4^{n}\left(\begin{array}{c}
n+t / 2-1 \\
n
\end{array}\right) x^{n}
$$

Since

$$
1+\frac{1}{g(x)}=\frac{2}{C(x)},
$$

we deduce that

$$
\prod_{i=1}^{r} g(x) C(x)^{-a_{i}}=g(x)^{r} C(x)^{-|\mathbf{a}|}=2^{-|\mathbf{a}|} g(x)^{r-|\mathbf{a}|}(1+g(x))^{|\mathbf{a}|} .
$$

Note that the left-hand side of (2) is the general term (i.e., the coefficient of the term in $x^{n}$ ) of the left-hand side of (5). Moreover, since the general term of $g(x)^{r-|\mathbf{a}|+j}$ is $4^{n}\left(\begin{array}{c}n+(r-|\mathbf{a}|+j) / 2-1 \\ n\end{array}\right)$, the right-hand side of (2) is the general term of the right-hand side of (5).

Hence, the new proof of Theorem 1 is complete.

Acknowledgments We kindly acknowledge the anonymous reviewer for valuable comments and for providing a new proof of Theorem 1.

Open Access This article is distributed under the terms of the Creative Commons Attribution License which permits any use, distribution, and reproduction in any medium, provided the original author(s) and the source are credited.

\section{References}

1. Abel, U.: A generalization of the Leibniz Rule. Amer. Math. Mon. 120(10), 924-928 (2013)

2. Duarte, R., Guedes de Oliveira, A.: Note on the convolution of binomial coefficients. J. Integer Seq. 16 (2013) (Article 13.7.6)

3. Duarte, R., Guedes de Oliveira, A.: A short proof of a famous combinatorial identity. arXiv:1307.6693. Accessed 30 October 2013 (preprint)

4. Chang, G., Xu, C.: Generalization and probabilistic proof of a combinatorial identity. Amer. Math. Mon. 118, 175-177 (2011)

5. Spivey, M.Z.: Alternating convolution of the central binomial coefficients. Amer. Math. Mon. 121, 537-540 (2014)

6. Spivey, M.Z.: Combinatorial proof that $\sum_{k=0}^{n}\left(\begin{array}{c}2 k \\ k\end{array}\right)\left(\begin{array}{c}2(n-k) \\ n-k\end{array}\right)(-1)^{k}=2^{n}\left(\begin{array}{c}n \\ n / 2\end{array}\right)$ is even (version: 2012-12-21). http://math.stackexchange.com/a/98327/ 